\title{
ON THE DISTRIBUTION OF THE MAXIMUM OF SUCCESSIVE CUMULATIVE SUMS OF INDEPENDENTLY BUT NOT IDENTICALLY DISTRIBUTED CHANCE VARIABLES
}

\author{
ABRAHAM WALD \\ 1. Introduction. Let $X_{1}, X_{2}, \cdots$, and so on be a sequence of chance \\ variables and let $S_{i}$ denote the sum of the first $i X$ 's, that is,

$$
S_{i}=X_{1}+\cdots+X_{i} \quad(i=1,2, \cdots, \text { ad inf }) .
$$

Let $M_{N}$ denote the maximum of the first $N$ cumulative sums $S_{1}, \cdots, S_{N}$, that is,

$$
M_{N}=\max \left(S_{1}, \cdots, S_{N}\right) .
$$

The distribution of $M_{N}$, in particular the limiting distribution of a suitably normalized form of $M_{N}$, has been studied by Erdös and Kac $[1]^{1}$ and by the author [2] in the special case when the $X$ 's are independently distributed with identical distributions.

In this note we shall be concerned with the distribution of $M_{N}$ when the $X$ 's are independent but not necessarily identically distributed. In particular, the mean and variance of $X_{i}$ may be any functions of $i$.

In \$2 lower and upper limits for $M_{N}$ are obtained which yield particularly simple limits for the distribution of $M_{N}$ when the $X$ 's are symmetrically distributed around zero.

In $\$ 3$ the special case is considered when $X_{i}$ can take only the values 1 and -1 but the probability $p_{i}$ that $X_{i}=1$ may be any function of $i$. The exact probability distribution of $M_{N}$ for this case is derived and expressed as the first row of a product of $N$ matrices.

The limiting distribution of $M_{N} / N^{1 / 2}$ is treated in $\$ 4$. Since the interesting limiting case arises when the mean of $X_{i}(i \leqq N)$ is not only a function of $i$ but also a function of $N$, we have to introduce a double sequence of chance variables. That is, for any $N$ we consider a sequence of $N$ chance variables $X_{N 1}, \cdots, X_{N N}$. Let $\mu_{N i}$ denote the mean and $\sigma_{N i}$ the standard deviation of $X_{N i}$. Let, furthermore, $S_{N i}$ denote the sum $X_{N 1}+\cdots+X_{N i}$ and $M_{N}$ the maximum of $S_{N 1}, \cdots$, $S_{N N}$. With the help of a method used by Erdös and Kac [1], the following theorem is established in $\$ 4$ : 1947.

Presented to the Society, September 4, 1947; received by the editors June 27,

1 Numbers in brackets refer to the references cited at the end of the paper. 
Theorem 1.1 Let $\left\{X_{N i}\right\}$ and $\left\{X_{N i}^{*}\right\}(i=1, \cdots, N ; N=1,2, \cdots$, ad inf.) be two sequences of chance variables such that the following conditions are fulfilled:

(a) The X's are independently distributed.

(b) The sequence $\left\{\sigma_{N i}\right\} \quad(i=1, \cdots, N ; N=1,2, \cdots$, ad inf.)

has a positive lower bound and a finite upper bound.

(c) $\mu_{N i} N^{1 / 2}$ is a bounded function of $i$ and $N$.

(d) The third absolute moment of $X_{N i}$ is a bounded function of $i$ and $N$.

(e) The conditions (a)-(d) remain valid if we replace $X_{N i}$ by $X_{N i}^{*}$.

(f) The equation

$$
\lim _{N=\infty}\left[\frac{\mu_{N 1}^{*}+\cdots+\mu_{N j_{i}}^{*}}{\sigma_{N 1}^{* 2}+\cdots+\sigma_{N j_{i}}^{* 2}}\right.
$$

$$
\left.-\frac{\mu_{N 1}+\cdots+\mu_{N i}}{\sigma_{N 1}^{2}+\cdots+\sigma_{N i}^{2}}\left(\frac{\sigma_{N 1}^{2}+\cdots+\sigma_{N N}^{2}}{\sigma_{N 1}^{* 2}+\cdots+\sigma_{N N}^{* 2}}\right)^{1 / 2}\right]=0
$$

holds for all $i$ and $N$ where $\mu_{N i}^{*}$ is the mean and $\sigma_{N i}^{*}$ is the standard deviation $X_{N i}^{*}$ and $j_{i}$ is the smallest positive integer for which

$$
\frac{\sigma_{N 1}^{* 2}+\cdots+\sigma_{N j_{i}}^{* 2}}{\sigma_{N 1}^{* 2}+\cdots+\sigma_{N N}^{* 2}} \geqq \frac{\sigma_{N 1}^{2}+\cdots+\sigma_{N i}^{2}}{\sigma_{N 1}^{2}+\cdots+\sigma_{N N}^{2}} .
$$

Let

$$
\bar{M}_{N}=M_{N}^{*}\left(\frac{\sigma_{N 1}^{2}+\cdots+\sigma_{N N}^{2}}{\sigma_{N 1}^{* 2}+\cdots+\sigma_{N N}^{* 2}}\right)^{1 / 2}
$$

where $M_{N}^{*}$ is the same function of the $X^{*}$ 's as $M_{N}$ is of the $X^{\prime}$ s. Then for any positive $\epsilon$ we have

(1.5) $\lim \inf \left[\operatorname{prob}\left\{M_{N}<c N^{1 / 2}\right\}-\operatorname{prob}\left\{\bar{M}_{N}<(c-\epsilon) N^{1 / 2}\right\}\right] \geqq 0$ and

(1.6) $\underset{N=\infty}{\lim \inf }\left[\operatorname{prob}\left\{\bar{M}_{N}<(c+\epsilon) N^{1 / 2}\right\}-\operatorname{prob}\left\{M_{N}<c N^{1 / 2}\right\}\right] \geqq 0$.

The following corollary is a simple consequence of Theorem 1.1:

CoROllary 1.1. Let $N^{\prime}$ be any positive integral valued and strictly increasing function of $N$ for which prob $\left\{\bar{M}_{N^{\prime}}<c N^{\prime 1 / 2}\right\}$ converges to a limit function $P(c)$ at all continuity points $c$ of $P(c)$ as $N \rightarrow \infty$. Then also 


$$
\lim _{N=\infty} \operatorname{prob}\left\{M_{N^{\prime}}<c N^{\prime 1 / 2}\right\}=P(c)
$$

at all continuity points $c$ of $P(c)$.

The validity of Corollary 1.1 can be derived from that of Theorem 1.1 as follows: Let $c=c_{0}$ be a continuity point of $P(c)$ and substitute $N^{\prime}$ for $N$ in (1.5) and (1.6). For any positive $\rho$ all limit points of prob $\left\{\bar{M}_{N^{\prime}}<\left(c_{0}-\epsilon\right) N^{\prime 1 / 2}\right\}$ and prob $\left\{\bar{M}_{N^{\prime}}<\left(c_{0}+\epsilon\right) N^{\prime 1 / 2}\right\}$ will lie in the interval $\left[P\left(c_{0}\right)-\rho, P\left(c_{0}\right)+\rho\right]$ for sufficiently small $\epsilon$. Hence, equations (1.5) and (1.6) imply that

$$
\begin{aligned}
P\left(c_{0}\right)-\rho & \leqq \liminf _{N=\infty} \operatorname{prob}\left\{M_{N^{\prime}}<c_{0} N^{\prime 1 / 2}\right\} \\
& \leqq \limsup _{N=\infty} \operatorname{prob}\left\{M_{N^{\prime}}<c_{0} N^{\prime 1 / 2}\right\} \leqq P\left(c_{0}\right)+\rho .
\end{aligned}
$$

Since (1.8) is true for any positive number $\rho$, Corollary 1.1 is proved.

The result in Corollary 1.1 can be expressed also by saying that for any subsequence $\left\{N^{\prime}\right\}$ of $\{N\}$ for which $\bar{M}_{N^{\prime}} / N^{\prime 1 / 2}$ has a limiting distribution as $N \rightarrow \infty$, also $M_{N^{\prime}} / N^{\prime 1 / 2}$ has a limiting distribution which is equal to that of $\bar{M}_{N^{\prime}} / N^{\prime 1 / 2}$.

It can easily be verified that the conditions (e) and (f) can always be satisfied for chance variables $X_{N t}^{*}$ which take only the values 1 and -1 with properly chosen probabilities. Thus, the results of $\$ 3$ may be used to compute

$$
\operatorname{prob}\left\{M_{N}^{*}<N^{1 / 2} c\left(\frac{\sigma_{N 1}^{* 2}+\cdots+\sigma_{N N}^{* 2}}{\sigma_{N 1}^{2}+\cdots+\sigma_{N N}^{2}}\right)^{1 / 2}\right\} .
$$

2. Derivation of upper and lower bounds for $M_{N}$. Let $X_{1}, \cdots, X_{N}$ be a set of $N$ variables and let

$$
\tilde{X}_{i}=X_{N-i+1} \quad(i=1,2, \cdots, N) .
$$

Let, furthermore,

$$
\begin{aligned}
\tilde{M}_{i}=\max \left(\tilde{X}_{i}, \tilde{X}_{i}+\tilde{X}_{i-1}, \cdots, \tilde{X}_{i}+\cdots\right. & \left.+\tilde{X}_{1}\right), \\
& (i=1, \cdots, N) .
\end{aligned}
$$

Clearly

$$
\tilde{M}_{N}=M_{N}=\max \left(X_{1}, X_{1}+X_{2}, \cdots, X_{1}+\cdots+X_{N}\right) .
$$

If $X_{1}, \cdots, X_{N}$ are independent chance variables, the chance variables $\tilde{M}_{1}, \tilde{M}_{2}, \cdots, \tilde{M}_{N}$ form a simple Markoff chain, that is, the conditional distribution of $\tilde{M}_{i+1}$, given $\tilde{M}_{1}, \cdots, \tilde{M}_{i}$, depends only 
on $\tilde{M}_{i}$. This is an immediate consequence of the relations:

$$
\tilde{M}_{i+1}=\tilde{M}_{i}+\tilde{X}_{i+1}
$$

if $\tilde{M}_{i}>0$

and

$$
\tilde{M}_{i+1}=\tilde{X}_{i+1}
$$

if $\tilde{M}_{i} \leqq 0$.

We shall now prove the following theorem:

ThEOREM 2.1. The inequality

$$
\tilde{M}_{i} \leqq\left|\epsilon_{1} \tilde{X}_{1}+\cdots+\epsilon_{i} \tilde{X}_{i}\right| \quad(i=1, \cdots, N)
$$

holds where $\epsilon_{1}=1, \epsilon_{i}=1$ if $\epsilon_{1} \tilde{X}_{1}+\cdots+\epsilon_{i-1} \tilde{X}_{i-1}>0$ and $\epsilon_{i}=-1$, if $\epsilon_{1} \tilde{X}_{1}+\cdots+\epsilon_{i-1} \tilde{X}_{i-1} \leqq 0$.

Proof. Clearly, (2.6) holds for $i=1$. We shall prove (2.6) for $i+1$ assuming that it holds for $i$. For this purpose it is sufficient to show, because of (2.4) and (2.5), that

$$
\left|\epsilon_{1} \tilde{X}_{1}+\cdots+\epsilon_{i+1} \tilde{X}_{i+1}\right|-\left|\epsilon_{1} \tilde{X}_{1}+\cdots+\epsilon_{i} \tilde{X}_{i}\right| \geqq \tilde{X}_{i+1} .
$$

Denote $\left|\epsilon_{1}, \tilde{X}_{1}+\cdots+\epsilon_{i} \tilde{X}_{i}\right|$ by $c_{i}$. If $c_{i}>0$, then $\epsilon_{i+1}=1$ and inequality (2.7) goes over into

$$
\left|c_{i}+\tilde{X}_{i+1}\right|-c_{i} \geqq \tilde{X}_{i+1}
$$

which is obviously true. If $c_{i} \leqq 0, \epsilon_{i+1}=-1$ and inequality (2.7) is equivalent with

$$
|| c_{i}\left|+X_{i+1}\right|-\left|c_{i}\right| \geqq X_{i+1}
$$

which is obviously true. Hence, Theorem 2.1 is proved.

We shall now prove a theorem giving a lower bound for $\tilde{M}_{i}$.

THEOREM 2.2. The inequality

$$
\tilde{K}_{i}=\left|\epsilon_{1} \tilde{X}_{1}+\cdots+\epsilon_{i} \tilde{X}_{i}\right|-2 \max _{j \leqq i}\left|\tilde{X}_{i}\right| \leqq \tilde{M}_{i}
$$

$$
(i=1, \cdots, N)
$$

holds where the $\epsilon$ 's are defined as in Theorem 2.1.

Proof. Theorem 2.2 is obviously true for $i=1$. We shall assume that it is valid for $i$ and we shall prove it for $i+1$. It follows from (2.4) and (2.5) that

$$
\begin{aligned}
\tilde{M}_{i+1}-\tilde{M}_{i} & \geqq \tilde{X}_{i+1}, \\
\tilde{M}_{i+1} & \geqq \tilde{X}_{i+1} .
\end{aligned}
$$


Hence, to prove (2.10) for $i+1$ assuming that it is true for $i$, it is sufficient to show that at least one of the following two inequalities holds:

$$
\begin{aligned}
\tilde{K}_{i+1}-\tilde{K}_{i} & \leqq \tilde{X}_{i+1}, \\
\tilde{K}_{i+1} & \leqq \tilde{X}_{i+1} .
\end{aligned}
$$

Consider first the case when $\left|\tilde{X}_{i+1}\right| \leqq\left|\epsilon_{1} \tilde{X}_{1}+\cdots+\epsilon_{i} \tilde{X}_{i}\right|$. In this case (2.13) always holds, as can easily be verified. If $\left|\tilde{X}_{i+1}\right|$ $>\left|\epsilon_{1} \tilde{X}_{1}+\cdots+\epsilon_{i} \tilde{X}_{i}\right|$ and $\tilde{X}_{i+1} \geqq 0$, then (2.13) holds again. If $\left|\tilde{X}_{i+1}\right|>\left|\epsilon_{1} \tilde{X}_{1}+\cdots+\epsilon_{i} \tilde{X}_{i}\right|$ and $\tilde{X}_{i+1}<0$, then $\mid \epsilon_{1} \tilde{X}_{1}+\cdots+\epsilon_{i} \tilde{X}_{i}$ $+\epsilon_{i+1} \tilde{X}_{i+1}|\leqq| \tilde{X}_{i+1} \mid$ and, therefore, $\widetilde{K}_{i+1} \leqq\left|X_{i+1}\right|-2 \max _{j \leqq i+1}\left|X_{j}\right|$ $\leqq-\left|\tilde{X}_{i+1}\right|=\tilde{X}_{i+1}$. Thus, in this case the inequality (2.14) holds. This completes the proof of Theorem 2.2.

Since $\tilde{M}_{N}=M_{N}$, Theorems 2.1 and 2.2 yield the following limits for $M_{N}$

$$
\begin{aligned}
\left|\epsilon_{1} \tilde{X}_{1}+\cdots+\epsilon_{N} \tilde{X}_{N}\right|-2 \max _{i \leqq N}\left|\tilde{X}_{i}\right| & \\
& \leqq M_{N} \leqq\left|\epsilon_{1} \tilde{X}_{1}+\cdots+\epsilon_{N} \tilde{X}_{N}\right| .
\end{aligned}
$$

Suppose now that $X_{1}, \cdots, X_{N}$ are chance variables such that the conditional distribution of $X_{i}(i=1, \cdots, N)$ for any given values of $X_{i+1}, \cdots, X_{N}$ is symmetric around the origin. Then the probability distribution of $\left|\epsilon_{1} \widetilde{X}_{1}+\cdots+\epsilon_{N} \widetilde{X}_{N}\right|$ is the same as that of $\left|X_{1}+\cdots+X_{N}\right|$, and the distribution of $\left|\epsilon_{1} \tilde{X}_{1}+\cdots+\epsilon_{N} \tilde{X}_{N}\right|$ $-2 \max _{i \leqq N}\left|\tilde{X}_{i}\right|$ equals that of $\left|X_{1}+\cdots+X_{N}-2 \max _{i \leqq N}\right| X_{i} \mid$. It then follows from (2.15) that the following theorem holds:

THEOREM 2.3. If the conditional distribution of $X_{i}(i=1,2, \cdots, N)$, for any given value of $X_{i+1}, \cdots, X_{N}$ is symmetric around the origin, the inequality

$$
\begin{aligned}
& \operatorname{prob}\left\{\left|X_{1}+\cdots+X_{N}\right|<c\right\} \leqq \operatorname{prob}\left\{M_{N}<c\right\} \\
& \leqq \operatorname{prob}\left\{\left|X_{1}+\cdots+X_{N}\right|-2 \max _{i \leqq N}\left|X_{i}\right|<c\right\}
\end{aligned}
$$

holds for any value $c$.

Inequality (2.15) has also some interesting implications for the asymptotic distribution theory of $M_{N}$. In most cases we shall be concerned with the limiting distribution of $M_{N} / N^{1 / 2}$ as $N \rightarrow \infty$ (this is the case discussed in $\$ 4)$. If $\left(1 / N^{1 / 2}\right) \max _{i \leqq N}\left|X_{i}\right|$ converges stochastically to zero, as will usually be the case, inequality $(2.15) \mathrm{im}$ plies that the limiting distribution of $M_{N} / N^{1 / 2}$ is the same as that of $\left(1 / N^{1 / 2}\right)\left|\epsilon_{1} \tilde{X}_{1}+\cdots+\epsilon_{N} \widetilde{X}_{N}\right|$. 
3. The distribution of $M_{N}$ when $X_{i}$ can take only the values 1 and -1 . Let $X_{1}, \cdots, X_{N}$ be independently distributed chance variables such that $X_{i}$ can take only the values 1 and -1 . Let $p_{i}$ denote the probability that $X_{i}=1$. The probability that $X_{i}=-1$ is then equal to $1-p_{i}=q_{i}$.

Let $\tilde{X}_{i}$ and $\tilde{M}_{i}(i=1, \cdots, N)$ be defined by (2.1) and (2.2), respectively. One can easily verify that $\tilde{M}_{i}$ can take only the values $-1,0,1,2, \cdots, i$. Let $c_{i j}$ denote the probability that $\tilde{M}_{i}=j$ for $j=1, \cdots, i$, and let $c_{i 0}$ be the probability that $\tilde{M}_{i} \leqq 0$. It follows from the definition of the $\tilde{M}$ 's that the following recursion formulas hold:

$$
\begin{gathered}
c_{i+1,0}=q_{i+1} c_{i 0}+q_{i+1} c_{i 1}, \\
c_{i+1, j}=p_{i+1} c_{i, j-1}+q_{i+1} c_{i, j+1} \quad(j=1,2, \cdots, i+1) .
\end{gathered}
$$

Since $\tilde{M}_{N}=M_{N}$, we have

$$
\begin{aligned}
& \operatorname{prob}\left\{M_{N}=j\right\}=c_{N} \quad \text { for } j=1, \cdots, N, \\
& \operatorname{prob}\left\{M_{N} \leqq 0\right\}=c_{N 0} .
\end{aligned}
$$

We shall now construct $N$ square matrices $A_{1}, \cdots, A_{N}$, each having $N+1$ rows and $N+1$ columns, such that the first row of the prod-

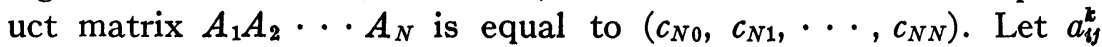
denote the element in the $i$ th row and $j$ th column of the matrix $A_{k}$ $(i, j=1, \cdots, N+1 ; k=1, \cdots, N)$. We put

$$
\begin{array}{r}
a_{11}^{k}=q_{k} ; \quad a_{i, i+1}^{k}=p_{k} \quad(i=1,2, \cdots, N) ; \\
a_{i, i-1}^{k}=q_{k} \quad(i=2,3, \cdots, N+1)
\end{array}
$$

and all other elements $a_{v j}^{k}$ equal to zero. It then follows easily from the recursion formulas (3.1) and (3.2) that the first row of the product $A_{1} A_{2} \cdots A_{N}$ is equal to $\left(c_{N 0}, c_{N 1}, \cdots, c_{N N}\right)$. Thus, the first row of the product $A_{1} A_{2} \cdots A_{N}$ yields the exact probability distribution of $M_{N}$.

Starting with the initial values $c_{10}=q_{1}, c_{11}=p_{1}, c_{1 j}=0$ for $j>1$, the final values $c_{N 0}, c_{N 1}, \cdots, c_{N N}$ can be best computed by repeated application of the recursion formulas (3.1) and (3.2).

4. Proof of Theorem 1.1. Let $\left\{X_{N i}\right\}$ and $\left\{X_{N i}^{*}\right\}$ be two double sequences of chance variables for which conditions (a)-(f) of Theorem 1.1 are fulfilled. Let $k$ be a positive integer and $N_{1}, \cdots, N_{k}$ a set of positive integers such that $N_{1}<N_{2}<\cdots<N_{k}=N$. Let, furthermore, 
(4.1) $\quad P_{N, k}(c)=\operatorname{prob}\left\{\max \left(S_{N N_{1}}, S_{N N_{2}}, \cdots, S_{N N_{k}}\right)<c N^{1 / 2}\right\}$.

Because of conditions (b) and (c) of Theorem 1.1, there exist two finite values $A$ and $B$ such that $A \geqq N \mu_{N,}^{2}$ and $B \geqq \sigma_{N i}^{2}$ for all $N$ and $i$. Let $\phi(k)$ be an upper bound of the values

$$
\frac{N_{1}}{N}, \frac{N_{2}-N_{1}}{N}, \cdots, \frac{N_{k}-N_{k-1}}{N} .
$$

For any positive $\epsilon$ the following inequality holds:

$$
P_{N, k}(c-\epsilon)-\frac{\phi(k)}{\epsilon^{2}}[B+A \phi(k)] \leqq P_{N}(c) \leqq P_{N, k}(c),
$$

where $P_{N}(c)=$ prob $\left\{M_{N}<c N^{1 / 2}\right\}$. Using a method given by Erdös and $\mathrm{Kac}[1]$, the author [2] has proved the above inequality when $\mu_{N i}=\mu_{N}, \sigma_{N i}=1$ and $N_{j}=[j N / k]$. To adapt the proof given in [2] to the more general case treated here, it is sufficient to replace the right-hand member of (2.6) in [2] by

$$
\frac{\left(N_{i+1}-N_{i}\right) B+\left(N_{i+1}-N_{i}\right)^{2} \mu_{N}^{2}}{\epsilon^{2} N},
$$

where $\mu_{N}^{2}=\max \left(\mu_{N 1}^{2}, \cdots, \mu_{N N}^{2}\right)$.

For the purpose of proving Theorem 1.1, we shall choose $N_{j}$ to be the smallest positive integer for which

$$
\sigma_{N 1}^{2}+\cdots+\sigma_{N N_{j}}^{2} \geqq \frac{j\left(\sigma_{N 1}^{2}+\cdots+\sigma_{N N}^{2}\right)}{k} .
$$

Since $\sigma_{N i}^{2}$ has a positive lower bound and a finite upper bound, there exists a positive constant $h$, independent of $k$, such that $h / k$ is an upper bound of the values (4.2). It then follows from (4.3) that

$$
P_{N, k}(c-\epsilon)-\frac{1}{\epsilon^{2} k}(a+b / k) \leqq P_{N}(c) \leqq P_{N, k}(c)
$$

when $a$ and $b$ are positive constants independent of $N, k, c$ and $\epsilon$.

Clearly, if Theorem 1.1 is true for the special case when $\sigma_{N 1}^{2}+\ldots$ $+\sigma_{N N}^{2}=\sigma_{N 1}^{* 2}+\cdots+\sigma_{N N}^{* 2}$, it must be true also in the general case. Hence, it is sufficient to prove Theorem 1.1 when $\sigma_{N 1}^{2}+\cdots+\sigma_{N N}^{2}$ $=\sigma_{N 1}^{* 2}+\cdots+\sigma_{N N}^{* 2}$. In what follows we shall therefore restrict ourselves to this special case.

Let $N_{j}^{*}, P_{N, b}^{*}(c)$, and $P_{N}^{*}(c)$ have the same meaning with reference to the $X^{*}$ 's as $N, P_{N, k}(c)$, and $P_{N}(c)$ with reference to the $X$ 's. Then we have 
(4.7) $\quad P_{N, k}^{*}(c-\epsilon)-\frac{1}{\epsilon^{2} k}\left(a^{*}+b^{*} / k\right) \leqq P_{N}^{*}(c) \leqq P_{N, k}^{*}(c)$,

where $a^{*}$ and $b^{*}$ are positive constants independent of $N, k, c$ and $\epsilon$.

Let $G_{k 1}^{N}, G_{k 2}^{N}, \cdots, G_{k \mathbf{k}}^{N}$ be independently and normally distributed chance variables and let the mean and standard deviation of $G_{\mathbf{k}}^{N}$ be equal to the mean and standard deviation of $(k / N)^{1 / 2}\left(S_{N N_{i}}-S_{N N_{i-1}}\right)$, respectively. Let, furthermore,

Clearly, the mean and standard deviation of $G_{k t}^{N}$ are bounded functions of $N, k$ and $i$. Furthermore, the standard deviation of $G_{k t}^{N}$ has a positive lower bound. It then follows from condition (d) and the central limit theorem that

$$
\lim _{N=\infty}\left[Q_{N, k}(c)-P_{N, k}(c)\right]=0 .
$$

Let $G_{k t}^{* N}$ and $Q_{N, k}^{*}(c)$ have the same meaning with reference to the $X^{* \prime}$ s as $G_{k l}^{N}$ and $Q_{N, k}(c)$ with reference to the $X^{\prime}$ 's. We then have

$$
\lim _{N=\infty}\left[Q_{N, k}^{*}(c)-P_{N, k}^{*}(c)\right]=0 .
$$

It follows from condition (f) of Theorem 1.1 that

$$
\lim _{N=\infty} E\left(G_{k i}^{N}-G_{k i}^{* N}\right)=0
$$

$$
\lim _{N=\infty} E\left[\left(G_{k i}^{N}\right)^{2}-\left(G_{k i}^{* N}\right)^{2}\right]=0
$$

Hence

$$
\lim _{N=\infty}\left[Q_{N, k}(c)-Q_{N, k}^{*}(c)\right]=0 .
$$

From (4.9) and (4.10) and (4.13) we obtain

$$
\lim _{N=\infty}\left[P_{N, k}(c)-P_{N, k}^{*}(c)\right]=0 .
$$

Equations (4.6) and (4.14) give

$$
\liminf _{N=\infty}\left[P_{N}(c)-P_{N, k}^{*}(c-\epsilon)+\frac{1}{\epsilon^{2} k}\left(a+\frac{b}{k}\right)\right] \geqq 0
$$

and 


$$
\underset{N=\infty}{\lim \inf }\left[P_{N, k}^{*}(c)-P_{N}(c)\right] \geqq 0
$$

Since

$$
P_{N, k}^{*}(c-\epsilon) \geqq P_{N}^{*}(c-\epsilon)
$$

and since, because of (4.7),

$$
P_{N, k}^{*}(c)-\frac{1}{\epsilon^{2} k}\left(a^{*}+b^{*} / k\right) \leqq P_{N}^{*}(c+\epsilon),
$$

we obtain from (4.15) and (4.16)

(4.19) $\quad \liminf _{N=\infty}\left[P_{N}(c)-P_{N}^{*}(c-\epsilon)+\frac{1}{\epsilon^{2} k}\left(a+\frac{b}{k}\right)\right] \geqq 0$

and

$$
\liminf _{N=\infty}\left[P_{N}^{*}(c+\epsilon)+\frac{1}{\epsilon^{2} k}\left(a^{*}+\frac{b^{*}}{k}\right)-P_{N}(c)\right] \geqq 0 .
$$

Hence, since $k$ can be chosen arbitrarily large, we obtain

$$
\lim _{N=\infty} \inf \left[P_{N}(c)-P_{N}^{*}(c-\epsilon)\right] \geqq 0
$$

and

$$
\lim _{N=\infty} \inf _{N}\left[P_{N}^{*}(c+\epsilon)-P_{N}(c)\right] \geqq 0 .
$$

This concludes the proof of Theorem 1.1. It may be of interest to note that (4.21) and (4.22) imply that for any subsequence $\left\{N^{\prime}\right\}$ of the sequence $\{N\}$ we have

$$
\begin{aligned}
\lim \inf P_{N^{\prime}}^{*}(c-\epsilon) & \leqq \liminf _{N=\infty} P_{N^{\prime}}(c) \leqq \limsup _{N=\infty} P_{N^{\prime}}(c) \\
& \leqq \lim _{N=\infty} \sup _{N=\infty} P_{N^{\prime}}^{*}(c+\epsilon) .
\end{aligned}
$$

\section{REFERENCES}

1. P. Erdös and M. Kac, On certain limit theorems of the theory of probability, Bull. Amer. Math. Soc. vol. 52 (1946) pp. 292-302.

2. A. Wald, Limit distribution of the maximum and minimum of successive cumulative sums of random variables, Bull. Amer. Math. Soc. vol. 53 (1947) pp. 142-153.

Columbia University 